\title{
The feasibility of a pragmatic distance-based intervention to increase physical activity in lung cancer survivors
}

\author{
C.J. Peddle-McIntyre PhD, Postdoctoral Research Fellow ${ }^{1}$ (D) \\ M.K. Baker PhD, Senior Lecturer ${ }^{2}$ | Y.C.G. Lee MBChB PhD, Professor ${ }^{3}$ | \\ D.A. Galvão PhD, Professor ${ }^{1}$ | P. Cormie PhD, Associate Professor ${ }^{1,4}$ | \\ V. Graham BA, Project Coordinator ${ }^{1}$ | R.U. Newton PhD, Professor ${ }^{1,5}$
}

\footnotetext{
${ }^{1}$ Exercise Medicine Research Institute, Edith Cowan University, Joondalup, WA, Australia

${ }^{2}$ School of Exercise Science, Australian Catholic University, Strathfield, NSW, Australia

${ }^{3}$ Respiratory Department, Sir Charles Gairdner Hospital, Nedlands, WA, Australia

${ }^{4}$ Institute for Health and Aging, Australian Catholic University, Melbourne, Vic., Australia

${ }^{5} \cup Q$ Centre for Clinical Research, The University of Queensland, Herston, QId, Australia
}

\section{Correspondence}

Carolyn J. Peddle-McIntyre, Exercise Medicine Research Institute, Edith Cowan University, Joondalup WA, Australia.

Email: c.mcintyre@ecu.edu.au

Funding information

Carolyn Peddle-McIntyre is supported by a Cancer Council of Western Australia Postdoctoral Research Fellowship. Prof. Lee is a National Health \& Medical Research Council (NHMRC) Career Development Fellow and receives research project grant funding from the NHMRC, New South Wales Dust Disease Board, Sir Charles Gairdner Research Advisory Committee, Westcare and the Cancer Council of Western Australia. Daniel A. Galvão is supported by a Cancer Council Western Australia Research Fellowship. This research was supported by the Faculty Research Grant Scheme at Edith Cowan University
The purpose of this study was to investigate the feasibility and preliminary efficacy of a pragmatic distance-based intervention designed to increase physical activity (PA) participation in lung cancer survivors. Fourteen lung cancer survivors were recruited via invitation from the State Cancer Registry to join a 12-week PA intervention of print materials paired with brief telephone follow-up. Outcome measures of feasibility, PA participation and quality of life $(\mathrm{Q} O \mathrm{~L})$ were assessed at baseline, post-intervention and follow-up via telephone interview. Eligibility, recruitment and attrition rates were $16 \%, 58 \%$ and $29 \%$ respectively. No adverse events were reported; however, pain scores worsened following the intervention (median change -3.6, IQR -8.0, 0.0). Average intervention adherence was $91 \%$ with low median ratings of participation burden (i.e., all items 1/7) and high trial evaluation (i.e., all items 7/7). Post-intervention, median change in self-reported moderate and vigorous PA was $84 \min (I Q R-22,188)$, and several domains of QoL improved. However, for both of these outcomes, improvements were not maintained at follow-up. Our findings suggest that this pragmatic distance-based intervention was safe, had good adherence rates, and indicate potential for improving short-term PA and QoL in lung cancer survivors. Additional strategies are needed to improve other indicators of feasibility, particularly recruitment, retention and long-term maintenance of improvements. Australian New Zealand Clinical Trials Registration: ACTRN12612000085875.

KEYWORDS

distance-based, lung cancer, physical activity, quality of life

\section{1 | INTRODUCTION}

Lung cancer is a leading cause of invasive cancer internationally and accounts for one in every five cancer deaths worldwide (Ferlay et al., 2015). Following treatment, survivors of lung cancer (i.e., patients from the point of diagnosis for the remainder of life; Denlinger et al., 2014) face many ongoing symptoms such as fatigue, loss of physical functioning, shortness of breath and decreased quality of life (QoL;
Balduyck, Hendriks, Lauwers, \& van Schil, 2007; Brunelli et al., 2007; Handyjr et al., 2002; Zieren, Muller, Hamberger, \& Pichlmaier, 1996). Research indicates that only $30 \%-40 \%$ of lung cancer survivors meet physical activity (PA) guidelines (Coups et al., 2009b; Granger, Denehy, McDonald, Irving, \& Clark, 2014). Survivors of lung cancer are significantly less active, exhibit lower neuromuscular strength, are in worse nutritional status, have more depressive symptoms, and report lower QoL than non-cancer controls (Granger, McDonald, et al., 2014). Over 
time, PA levels decline, contributing to reductions in functional capacity and worsening symptoms (Granger, McDonald, et al., 2014).

Physical activity could play a key role in maintaining health and QoL for lung cancer survivors. Evidence suggests that lung cancer survivors 1-6 years post-surgery meeting PA guidelines (i.e., $150 \mathrm{~min}$ of moderate PA per week) report significantly higher QoL, less fatigue, shortness of breath and depressive symptoms than those not meeting guidelines (Coups et al., 2009a). In addition, lung cancer survivors often suffer with comorbid disease and are at a high risk of developing a recurrence of cancer (Aarts et al., 2015; Gester, Paulus, Sibille, Duysinx, \& Louis, 2016). PA could play a key role in maintaining functional independence as well as fitness to withstand future treatment for this population (Lakoski, Eves, Douglas, \& Jones, 2012).

Following lung resection, short-term supervised exercise interventions have demonstrated effectiveness for improving exercise capacity (Cavalheri, Tahirah, Nonoyama, Jenkins, \& Hill, 2014). However, supervised interventions have challenges for translation including financial costs, accessibility and the exclusion of survivors residing outside metropolitan areas (Peddle-Mcintyre, Bell, Fenton, McCargar, \& Courneya, 2012). In addition, these programmes are typically targeted at the immediate post-surgical population. The majority of long-term lung cancer survivors (55\%) report unmet supportive care needs regarding exercise and weight control (Yun et al., 2013). Given the potential benefits of PA, coupled with poor current participation rates but high interest levels, there is a clear need for interventions to increase PA levels for lung cancer survivors.

Interventions utilising print materials and telephone follow-up in cancer survivors have demonstrated positive effects for increasing PA levels, and self-reported physical functioning but are lacking in lung cancer (Goode, Lawler, Brakenridge, Reeves, \& Eakin, 2015). Similarly, interventions using a theoretical framework, such as the Theory of Planned Behavior, have been shown to be particularly effective for impacting behaviour change (Vallance, Courneya, Plotnikoff, \& Mackey, 2008). However, the majority of research to date has been conducted in breast cancer survivors (Goode et al., 2015). There are important differences between these cancer survivor populations that could impact the feasibility and fidelity of distance-based interventions. Lung cancer survivors have higher rates of comorbidities, particularly cardiovascular disease and respiratory disease, compared with other cancer survivor groups (Ogle, 2000). Lung cancer patients presenting for surgery have poor functional capacity (Handy et al., 2002), which is further compounded by specific side effects of lung resection such as decreased lung function and cardiorespiratory fitness (Khuri, McKenna, \& Movas, 2000). Research regarding tailored interventions that address specific functional, psychosocial, disease-related issues faced by lung cancer survivors is required.

The majority of early-stage lung cancer survivors report a desire and willingness to engage in PA and would prefer a programme tailored to lung cancer survivors (Philip et al., 2014). Walking has been reported as the preferred PA modality (Philip et al., 2014), and homebased activity is also a preferential location for training among lung cancer survivors (Leach, Devonish, Bebb, Krenz, \& Culos-Reed, 2015). Offering survivors a low-burden intervention aimed at the most preferred modality of PA with information specifically tailored to lung cancer survivors could be feasible and effective in increasing PA as well as QoL in this population.

Therefore, we conducted a prospective single group pilot study to investigate the feasibility of a distance-based intervention designed to increase PA levels in lung cancer survivors. Our primary aim was to examine feasibility indicators (i.e., eligibility rate, recruitment rate, attrition rate, adherence, number of adverse events, ratings of burden and acceptability). Our secondary aim was to describe the effects of the intervention on PA participation and patient-reported outcomes of QoL, fatigue and shortness of breath. We hypothesised that the intervention would be feasible and result in increases in PA and improvements in patient-reported outcomes at post-intervention and follow-up.

\section{2 | METHODS}

\section{1 | Setting and participants}

The study was conducted at the Exercise Medicine Research Institute at Edith Cowan University, Perth, Western Australia. Ethical approval was granted from the Edith Cowan University Research Ethics Committee and the Department of Health Western Australia Human Research Ethics Committee. Informed consent was obtained from all participants. Eligibility criteria included confirmed stage I-IIIB nonsmall-cell lung cancer, or limited stage small cell lung cancer; not meeting exercise guidelines (i.e. performing $<150$ min of moderate and vigorous PA per week); completed treatment for cancer for $\geq 8$-weeks; have no planned cancer treatment in the next 12 weeks; ability to read and understand English; access to a telephone; over 18 years of age; and no musculoskeletal, neurological, or cardiovascular disorder that could inhibit exercise or put a participant at risk of injury or illness.

\section{2 | Design and recruitment}

Survivors were recruited via mailout invitation through the Western Australia State Cancer Registry. We conducted a prospective single group feasibility study. Potentially eligible participants were identified through the Cancer Registry and sent a recruitment package containing information about the study including a study response form, researchers' contact information and a pre-paid envelope. Participants interested in the study were requested to contact the researchers by telephone, email, or via study response form in a pre-paid envelope. A reminder letter was mailed 3 weeks later if no contact was made with the Cancer Registry or researchers. Potential participants were subsequently screened via telephone for eligibility and enrolled if appropriate.

\section{3 | Physical activity intervention}

The PA intervention consisted of print materials paired with telephone follow-up designed to increase PA. The intervention was delivered over 12 weeks according to the schedule outlined in Figure 1. The goal was to progress towards achieving 150 min of moderate PA per week, which is the current aerobic exercise recommendations for cancer survivors (Schmitz et al., 2010). The intervention focused on 


\begin{tabular}{|c|c|c|c|c|c|c|c|c|c|c|c|c|c|c|}
\hline \multirow{2}{*}{ Week } & \multirow{2}{*}{$\begin{array}{c}\text { Baseline } \\
0\end{array}$} & \multicolumn{11}{|c|}{ Intervention } & \multirow{2}{*}{$\begin{array}{c}\text { Post } \\
13\end{array}$} & \multirow{2}{*}{$\begin{array}{c}\text { Follow-up } \\
24\end{array}$} \\
\hline & & 1 & 2 & 3 & 4 & 5 & 6 & 7 & 8 & 9 & 10 & 11 & & \\
\hline Intervention & $\Delta$ & * & * & * & * & & * & & * & & * & * & $\Delta$ & $\Delta$ \\
\hline
\end{tabular}

FIGURE 1 Intervention schedule

TABLE 1 Overview of print-material intervention components

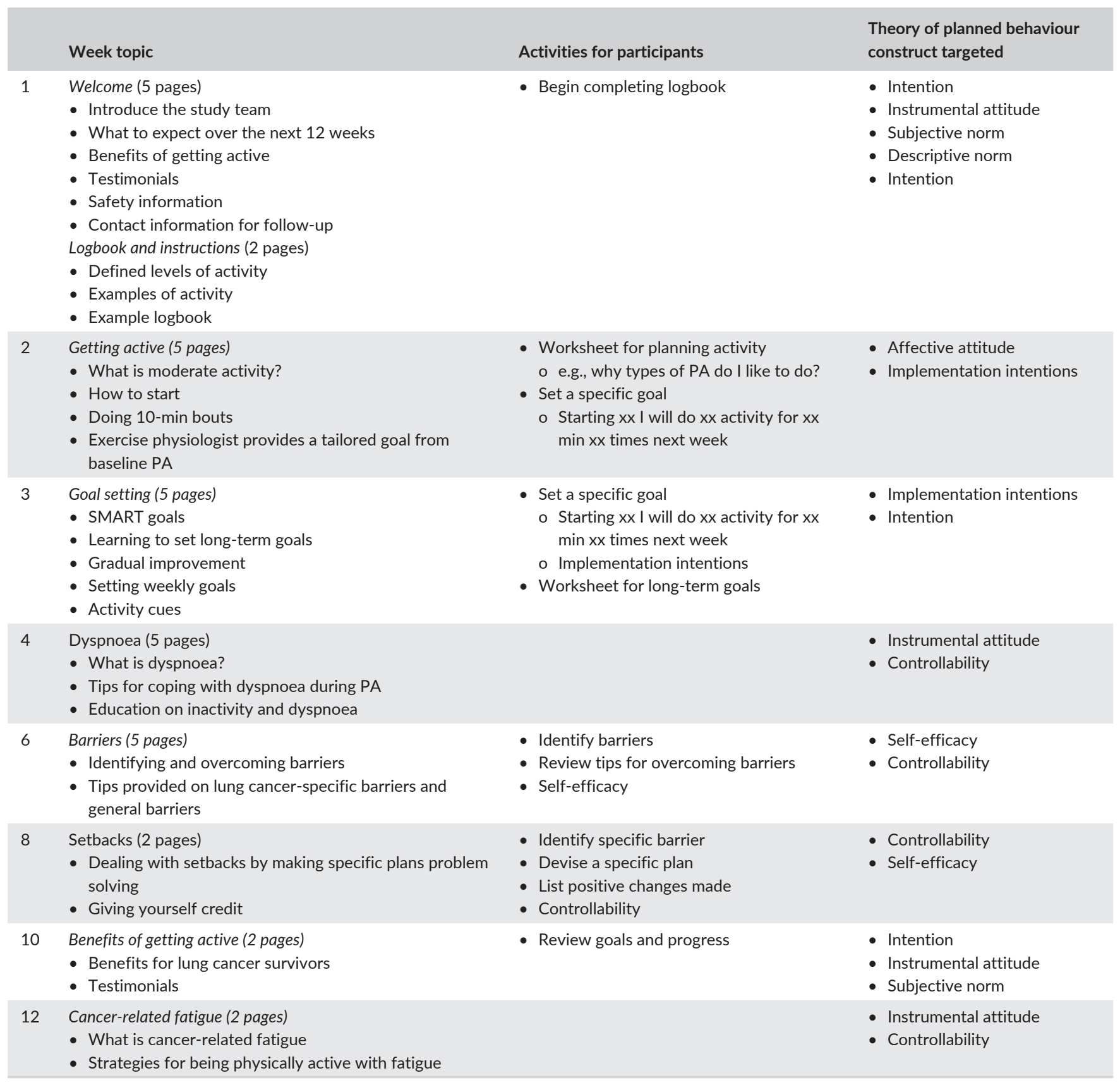

increasing walking, which constitutes the majority of daily PA and is the most common, and preferred, form of PA among lung cancer survivors (Granger, Denehy, et al., 2014).
An overview of the components of the print materials is outlined in Table 1. The materials were developed through literature review, including previous work from breast cancer survivors (Vallance, Courneya, 
Taylor, Plotnikoff, \& Mackey, 2008). The intervention print materials were developed in accordance with the framework of the Theory of Planned Behavior (Ajzen, 1991). The intervention targeted the theoretical constructs of intention, instrumental and affective attitude, subjective and descriptive norms, as well as self-efficacy and controllability as outlined in Table 1 (Vallance, Courneya, Plotnikoff, et al., 2008). The intervention used previously elicited attitudes, beliefs, norms and barriers in lung cancer survivors to inform the development of materials (Peddle et al., 2009; Peddle-Mcintyre, Bell, Fenton, McCargar, \& Courneya, 2013). In addition, the print materials were designed to address specific functional, psychosocial, disease-related issues faced by lung cancer survivors. Print materials provided contact information to access a telephone consultation with an exercise physiologist with expertise in lung cancer.

The Edith Cowan University Survey Research Centre conducted the telephone follow-up. This Centre is experienced in performing telephone follow-up assessing lifestyle (Gunnell et al., 2013). Telephone calls were designed to last between 5 and $10 \mathrm{~min}$. Each scripted call was tailored to compliment the print materials. At each contact, survivors were asked what barriers to PA were faced that week, what activities they performed, what they planned to do in the following week, how much moderate PA they recorded in their logbook, and if they experienced any adverse events. In addition, survivors were given a brief overview (i.e., the take-home message) of the topic for the week (e.g., fatigue), and asked if they would like any additional assistance (i.e., a call from an exercise physiologist). Staff conducting telephone follow-up received two sessions of training regarding the clinical population and implementing the intervention.

\section{4 | Outcome measures}

All outcome measures were assessed by telephone interview using Surveycraft (IBM ${ }^{\circledR}$ SPSS $^{\circledR}$ Surveycraft ${ }^{\mathrm{TM}}$ ), which is a Computer Assisted Telephone interview programme, and completed at baseline (week 0), post-intervention (week 13) and follow-up (week 24). Medical and demographical information was collected during screening via telephone interview.

\subsection{1 | Feasibility outcomes}

Feasibility outcomes included eligibility rate, recruitment rate, attrition rate, adherence and number of adverse events. Specifically, eligibility rate was defined as the total number of participants eligible as a percentage of the total number approached. Recruitment rate was defined as the number of participants recruited as a percentage of the total number eligible. Attrition rate was defined as number of participants lost to follow-up as a percentage of the total number enrolled (Sellar et al., 2014). Adherence was defined as the number of telephone follow-up calls attended out of a possible eight, for all participants regardless of attrition. At each telephone contact, participants were asked to report adverse events.

Acceptability of the intervention was measured by assessment of ratings of burden and acceptability. At post-intervention, survivors were asked to rate the burden of (1) receiving the telephone calls, (2) reviewing the print materials, (3) completing the questionnaires, (4) doing the home-based PA and (5) completing the logbook. Survivors were also asked to rate the acceptability of the trial in regard to participation being: (1) a waste of my time, (2) useful research for helping others, (3) useful for me personally, (4) something I would recommend to other lung cancer survivors and (5) something that will help me continue exercising on my own. Each item was scored from 1 (not at all) to 7 (very much; Peddle-Mcintyre et al., 2012; Rogers, Markwell, Courneya, McAuley, \& Verhulst, 2011). To assess acceptability of the intervention materials, at each telephone contact, survivors were asked: (1) if the material was relevant, (2) if the material was easy to understand and (3) if they attempted to do the activity (in weeks that there was a written activity included in the print material).

\subsection{2 | Physical activity level}

Patient-reported PA was measured by the Godin Leisure Time Exercise Questionnaire (Godin, Jobin, \& Bouillon, 1986). Moderate and vigorous physical activity (MVPA) was calculated by combining minutes with a double weighting on vigorous intensity minutes (Armstrong, Bauman, \& Davies, 2000). Meeting the intervention target was defined as those self-reporting 150 min of MVPA at post-intervention (Schmitz et al., 2010).

\subsection{3 | Patient-reported outcomes}

General and disease-specific QoL was assessed by The Medical Outcomes Study Short-Form 36 (Jenkinson, Wright, \& Coulter, 1994; Lyons, Perry, \& Littlepage, 1994) and the Functional Assessment of Cancer Therapy (FACT)-Lung Cancer Subscale (Cella et al., 1995), respectively. Cancer-specific symptoms of fatigue (FACT-Fatigue; Cella, Eton, Lai, Peterman, \& Merkel, 2002) as well as shortness of breath (Cancer Dyspnoea Scale; Tanaka, Akechi, Okuyama, Nishiwaki, \& Uchitomi, 2000) were also measured. Demographical and medical characteristics were collected via self-report. Participants were asked to answer yes/no to a list of questions (e.g., have you been told you have diabetes, have you been told you have high blood pressure), additionally participants were asked to list all illnesses or health conditions. Number of comorbidities was defined as the total number of self-reported conditions. Participants were asked to list all medications that were currently being undertaken. Number of medications was defined as the total number of medications reported.

\section{5 | Statistical analysis}

All analyses were conducting using PASW Statistics 23.0 (IBM Inc., Somers, NY). Differences between responders and non-responders to our mailout were assessed using independent samples $t$-test and chisquare. As this was a pilot feasibility study, one of the study goals was to gain information to inform sample size calculations to adequately power future randomised controlled trials, paired with the fact that no current clinically meaningful difference has been established for PA participation in this population, we did not perform an a priori sample 
150 post-treatment stage I-III lung cancer survivors identified

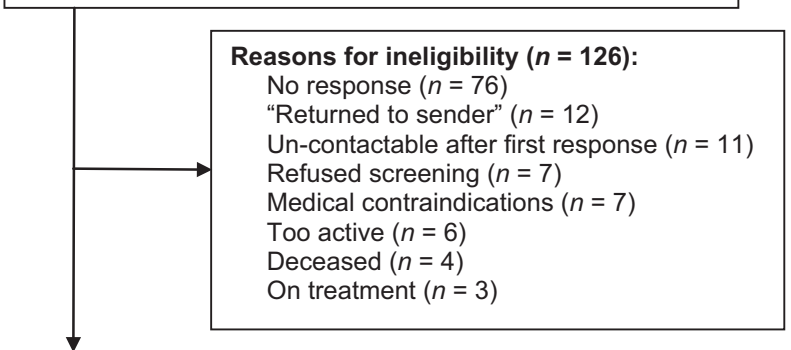

24 lung cancer survivors eligible

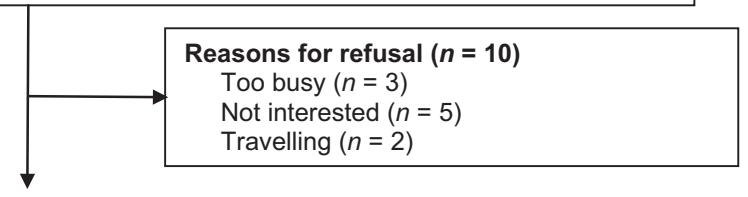

14 lung cancer survivors enrolled in the physical activity intervention

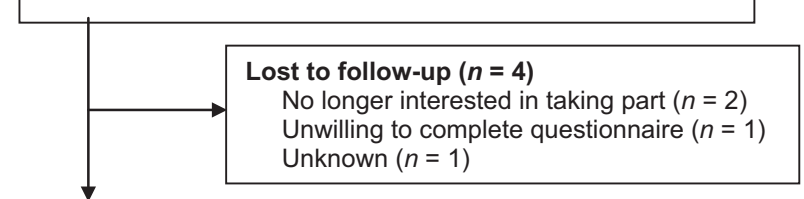

10 lung cancer survivors completed post-intervention assessment (week 13)

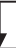

10 lung cancer survivors completed follow-up assessment (week 24)

FIGURE 2 Flow of participants through the trial

size calculation. Descriptive statistics (i.e., mean and standard deviation) were used to describe feasibility outcomes as well as medical and demographical characteristics. Given that this was a feasibility study with a limited sample size and lack of normal distribution in several outcomes, median and interquartile range were reported for PA level and patientrated outcomes at each time point. Median change score and interquartile range are presented for each pairwise comparison for all complete cases between time points. Significance was defined as a $p<.05$.

\section{3 | RESULTS}

Participant flow through the trial is outlined in Figure 2. Between June and September 2012, 150 post-treatment lung cancer survivors were mailed the recruitment package. To assess the representativeness of our sample, we compared those that were eligible $(n=24)$ and those not eligible for the intervention $(n=126)$ based on the limited medical and demographical variables available from the cancer registry. Eligible and non-eligible participants did not differ in terms of age at mailout ( 67.2 years vs. 68.9 years; $p=.472$ ), age at diagnosis (62.7 years vs. 63.8 years; $p=.649$ ), years since diagnosis ( 4.5 years vs. 5.1 years; $p=.303$ ), or sex ( $46 \%$ male vs. $52 \%$ male; $p=.257$ ).
TABLE 2 Baseline demographical, medical of lung cancer survivors

\begin{tabular}{|c|c|c|c|}
\hline Variable & $N$ & & $\%$ \\
\hline Age, mean (range) years & & $68(42-91)$ & \\
\hline Sex, female & 6 & & 43 \\
\hline Number of medications, mean (SD) & & $3.1(2.1)$ & \\
\hline Number of comorbidities, mean (SD) & & $1.9(1.1)$ & \\
\hline Hypertension & 9 & & 64 \\
\hline Anxiety/depression & 4 & & 29 \\
\hline Osteoporosis & 3 & & 21 \\
\hline Diabetes & 3 & & 21 \\
\hline Gout & 2 & & 14 \\
\hline High cholesterol & 2 & & 14 \\
\hline Emphysema & 1 & & 7 \\
\hline Rheumatoid arthritis & 1 & & 7 \\
\hline Kidney disease & 1 & & 7 \\
\hline Thyroid disease & 1 & & 7 \\
\hline $\begin{array}{l}\text { Baseline MRC dyspnoea score, } \\
\text { median (IQR) }\end{array}$ & & $1.57(0.76)$ & \\
\hline Time since diagnosis, mean (range) year & & $3.7(1-7)$ & \\
\hline $\begin{array}{l}\text { Time since treatment completion, } \\
\text { mean (range) year }{ }^{a}\end{array}$ & & $3.4(1-7)$ & \\
\hline Diagnosis: NSCLC & 14 & & 100 \\
\hline \multicolumn{4}{|l|}{ Treatment } \\
\hline Surgery & 11 & & 73 \\
\hline Adjuvant chemotherapy & 2 & & 14 \\
\hline Chemo/RT & 4 & & 29 \\
\hline
\end{tabular}

NSCLC, non-small-cell lung cancer; MRC, Medical Research Council. ${ }^{a}$ Data were available by year (i.e., year of treatment completion).

Medical and demographical characteristics are reported in Table 2. Participants had a mean age of 68 , the majority had early-stage lung cancer (73\%), had received surgery (73\%), were male $(57 \%)$ and had hypertension (64\%).

\section{1 | Feasibility}

The eligibility and recruitment rate for the study were $16 \%$ and $58 \%$ respectively. Of the 14 participants enrolled, $50 \%$ lived in rural Western Australia. The attrition rate of enrolled participants was $29 \%$. The average adherence to the telephone follow-up was $91 \%$ (SD $=21 \%$; range $25 \%-100 \%)$. Eleven participants (79\%) completed the entire intervention and 12 participants completed at least $85 \%$ of the intervention calls.

\section{2 | Acceptability}

Among participants who completed post-testing, median ratings of burden were low (i.e., median for all items 1/7), and trial evaluations were high (i.e., median for all items 7/7). Over the course of the intervention, participants reported finding the materials relevant 
TABLE 3 Effects of distance-based physical activity intervention on patient-reported outcomes in lung cancer survivors

\begin{tabular}{|c|c|c|c|c|c|c|c|c|c|c|c|c|}
\hline & \multicolumn{2}{|c|}{ Baseline $(n=14)$} & \multicolumn{2}{|c|}{ Post $(n=10)$} & \multicolumn{2}{|c|}{$\begin{array}{l}\text { Follow-up } \\
(n=10)\end{array}$} & \multicolumn{2}{|c|}{$\begin{array}{l}\text { Baseline to post- } \\
\text { intervention }(n=10)\end{array}$} & \multicolumn{2}{|c|}{$\begin{array}{l}\text { Post-intervention to } \\
\text { follow-up }(n=10)\end{array}$} & \multicolumn{2}{|c|}{$\begin{array}{l}\text { Baseline to follow-up } \\
(n=10)\end{array}$} \\
\hline & Median & IQR & Median & IQR & Median & IQR & $\begin{array}{l}\text { Median } \\
\text { change }\end{array}$ & IQR & $\begin{array}{l}\text { Median } \\
\text { change }\end{array}$ & IQR & $\begin{array}{l}\text { Median } \\
\text { change }\end{array}$ & IQR \\
\hline \multicolumn{13}{|l|}{ Physical activity } \\
\hline Light Min & 82 & 129 & 144 & 147 & 121 & 133 & 93 & $-7.5,160$ & -10 & $-215,129$ & 52 & $-15,156$ \\
\hline \multicolumn{13}{|l|}{ QoL, SF-36 } \\
\hline $\begin{array}{l}\text { Physical } \\
\text { functioning }\end{array}$ & 46.0 & 17.3 & 49.7 & 10.0 & 48.6 & 13.2 & 1.1 & $-2.1,13.7$ & -2.1 & $-11.6,4.2$ & -1.0 & $-6.8,8.9$ \\
\hline Role functioning & 49.5 & 13.5 & 50.7 & 12.3 & 47.5 & 18.4 & 6.1 & $-5.5,9.8$ & -3.7 & $-8.6,1.2$ & 1.2 & $-11.0,5.5$ \\
\hline Bodily pain & 58.7 & 17.7 & 52.8 & 16.7 & 52.8 & 12.2 & -3.6 & $-8.0,0$ & 0.0 & $-8.1,8.0$ & 0.0 & $-12.0,1.1$ \\
\hline Role emotional & 45.0 & 7.7 & 46.5 & 0.0 & 46.5 & 6.2 & 0.0 & $0,3.9$ & 0.0 & $-6.2,0$ & 0.0 & $-1.6,3.8$ \\
\hline Mental health & 55.6 & 14.1 & 59.9 & 14.1 & 58.5 & 8.5 & 0.0 & $0,5.6$ & 0.0 & $-3.5,0.7$ & 0.0 & $-5.6,5.6$ \\
\hline PCS & 50.5 & 15.1 & 51.1 & 15.0 & 48.9 & 20.6 & 0.2 & $-2.3,6.2$ & -3.5 & $-7.4,2.5$ & 0.6 & $-9.0,4.6$ \\
\hline MCS & 50.1 & 15.9 & 53.0 & 8.0 & 52.9 & 20.1 & 1.5 & $-0.6,1.6$ & 0.1 & $-3.8,2.6$ & 0.7 & $-2.9,3.2$ \\
\hline \multicolumn{13}{|l|}{ QoL, FACT } \\
\hline FACT-G & 92.0 & 20.0 & 96.0 & 18.3 & 87.5 & 18.0 & 1.5 & $-5.0,7.3$ & -3.0 & $-9.0,2.5$ & -1.0 & $-8.5,3.0$ \\
\hline LCS & 27.0 & 9.3 & 26.4 & 4.8 & 26.5 & 9.5 & 3.0 & $0.6,4.0$ & -0.5 & $-4.0,1.0$ & 2.0 & $-0.8,4.3$ \\
\hline Fatigue & 44.5 & 10.3 & 45.0 & 16.0 & 43.0 & 15.5 & 1.5 & $-3.3,4.0$ & 0.5 & $-2.75-1.25$ & 1.0 & $-5.0,4.3$ \\
\hline
\end{tabular}

IQR, interquartile range; min, minutes; MVPA, moderate and vigorous physical activity; QoL, quality of life; SF-36, Short-Form 36; FACT, Functional Assessment of Cancer Therapy; LCS, Lung Cancer Subscale.

(median $=81 \%$ of the time; IQR $78 \%, 90 \%$ ) and easy to understand (median $=97 \%$ of the time, IQR $92 \%, 100 \%$ ). The majority of the time participants indicated attempting to complete the worksheets provided $($ median $=77 \%$, IQR 60\%-87\%).

\section{3 | PA level}

At baseline, 50\% of participants reported not taking part in any PA. Descriptive statistics of changes in PA level are reported in Table 3. MVPA increased from baseline to post-intervention (median change $=84 \mathrm{~min} /$ week) and decreased from post-intervention to follow-up (median change $=-60 \mathrm{~min} /$ week). The median change from baseline to follow-up was $0 \mathrm{~min} /$ week of MVPA. Light PA increased from baseline to post-intervention (median change $=93 \mathrm{~min} /$ week) and decreased from post-intervention to follow-up (median change $=-10 \mathrm{~min} /$ week). The median change from baseline to followup was $52 \mathrm{~min} /$ week of light activity.
Post-intervention, 54\% of participants reported increased levels of PA. Thirty-six per cent of participants reported achieving the programme target of 150 min of MVPA per week. The remaining $18 \%$ increased their activity level (i.e., increased from sedentary to insufficiently active between 1 and 149 min of MVPA). At follow-up, 20\% of participants reported achieving the programme target of $150 \mathrm{~min}$ of MVPA. Ten per cent of participants reported PA levels between 1 and 149 min of MVPA.

\section{4 | Patient-reported outcomes}

Descriptive statistics of changes in patient-reported outcomes are outlined in Table 3. From baseline to post-intervention, the median change in several domains of QoL reached the cut-point to be considered clinically meaningful (i.e., >3 points; Ware et al., 2007). Specifically, following the intervention, survivors reported improvements in domains of role functioning (median change $=6.1$ ), general 
health (median change $=4.8$ ), as well as lung cancer-specific aspects of QoL (median change $=3.0$ ). Bodily pain worsened from baseline to post-intervention (median change $=-3.6$ ). From post-intervention to follow-up, QoL declined in role functioning (median change $=-3.7$ ) and vitality (median change $=-3.1$ ). There were no meaningful changes in median QoL scores from baseline to follow-up. No other aspects of QoL or dyspnoea changed meaningfully at any time point.

\section{4 | DISCUSSION}

Here, we present the results of a pragmatic distance-based intervention in lung cancer survivors designed to increase PA. To our knowledge, this is the first distance-based PA intervention tested in lung cancer survivors. The study suffered from a low eligibility rate, and a $29 \%$ attrition rate, and a small sample size that may affect the generalisability of results. Intervention adherence rates were high, and participants reviewed the programme favourably. There were no adverse events reported, which lends support to the safety of this type of intervention in lung cancer survivors. However, participants reported a clinically meaningful worsening of bodily pain post-intervention. We found improvements in PA participation as well as some aspects of QoL at post-intervention, but these preliminary benefits were not sustained at follow-up. Importantly, our intervention included $50 \%$ of lung cancer survivors from a rural setting in Australia.

Overall, our intervention was shown to meet some, but not all indicators of feasibility. The eligibility rate $(16 \%)$ and recruitment rate (58\%) of the current intervention compare favourably with previous distance-based PA interventions in cancer survivors, they are low. Previous distance-based PA intervention research that employed cancer registries for recruitment report eligibility rates between $10 \%$ and 25\% (Demark-Wahnefried et al., 2006, 2007). Study reach, or recruitment rates, of distance-based PA and diet interventions vary widely with reported rates between $24 \%$ and $89 \%$ (Goode et al., 2015). The current eligibility rate is low and limits the generalisability of the findings of this research.

We report an attrition rate of $29 \%$ and an average intervention adherence of $91 \%$. However, this attrition rate is higher than that of a home-based walking intervention in lung cancer survivors (16\% and 24\%, respectively; Chen, Tsai, Wu, Lin, \& Lin, 2015). Distance-based PA interventions in other cancer survivor populations report attrition rates of 5\%-20\% following 12- to 14-week interventions (Bennett, Lyons, Winters-Stone, Nail, \& Scherer, 2007; Hatchett, Hallam, \& Ford, 2013; Ligibel et al., 2012; Pinto, Frierson, Rabin, Trunzo, \& Marcus, 2005; Vallance, Courneya, Plotnikoff, Yasui, \& Mackey, 2007). However, this research was largely conducted in breast, prostate or colorectal cancer survivors. Survivors of lung cancer suffer with greater comorbidity burden and decline in physical functioning (Leach, Bellizzi, Hurria, \& Reeve, 2016; Vijayvergia, Shah, \& Denlinger, 2015), which could make it more difficult to complete PA interventions. In this study, the main reason for drop-out given by participants was no longer wanting to receive telephone follow-up. In addition, one participant completed the entire intervention but refused to complete post-intervention/ follow-up questionnaires. This suggests that participants who dropped out might have found the intervention and outcome assessment to be burdensome.

Following the intervention, participants reported an average increase of $84 \mathrm{~min} /$ week in MVPA, which returned to baseline at follow-up. Meta-analysis of studies in cancer survivors suggests that broad-reach modalities, such as the ones employed here, are effective for improving PA (Goode et al., 2015). Distance-based PA interventions in breast, colon and rectal cancer survivors ranging from 12 to 16 weeks long report increases in PA ranging from 12 to $71 \mathrm{~min} /$ week (Ligibel et al., 2012; Pinto et al., 2005; Vallance et al., 2007). Research has indicated that lung cancer survivors report very low levels of MVPA (Coups et al., 2009b; Granger, Denehy, et al., 2014). The current results suggest that participants were accumulating over two additional sessions of MVPA per week following our PA intervention. While this increase in PA could result in meaningful improvements in health outcomes for this clinical population, currently there is no research available to define a clinically meaningful improvement in PA participation. However, current international and national exercise guidelines/recommendations are for all cancer survivors (including lung cancer) to accumulate $150 \mathrm{~min}$ of aerobic PA per week (Brown et al., 2003; Schmitz et al., 2010). As a result, increases of $\sim 80 \mathrm{~min}$ of PA in this group of patients are likely to be clinical meaningful but require further validation. Larger, adequately powered randomised trials are needed to determine what change in PA participation is required to achieve a clinically meaningful improvement in health outcomes and QoL in this population.

Overall, only about one-third of participants were able to achieve the programme goal of 150 min of MVPA following the intervention. A systematic review reports that adherence to PA goals in behavioural interventions in colorectal cancer survivors ranges from $13 \%$ to $47 \%$ (McCahon et al., 2015). Considering the medical challenges faced by this study population, paired with a very low baseline participation in PA (i.e., 50\% reported being sedentary at baseline), the intervention target could have been too challenging. A more modest intervention target that included goals for breaking up sedentary time and increasing light PA could have been more accessible to this patient group while still providing beneficial outcomes (Arem et al., 2015).

The majority of evidence suggests that PA is difficult to maintain during follow-up periods of distance-based interventions that employ telephone and/or print-based materials (Goode et al., 2015), with only a couple of studies reporting significant maintenance effects (Pinto, Papandonatos, Goldstein, Marcus, \& Farrell, 2013; Pinto et al., 2008). In fact, we reported that the increase in median MVPA reported following the intervention was not maintained at follow-up. It is possible that lung cancer survivors require support for a longer period than 12 weeks to retain improvements in PA participation.

In this study, we provide preliminary data to support a benefit of this programme in the QoL domains of general health, role functioning, and lung cancer-specific concerns, warranting a confirmation in future studies. Contrary, there was also a clinically meaningful worsening of bodily pain. Pain is a common symptom in lung cancer survivors; with almost $60 \%$ of those $1-6$ years post-treatment reporting pain (Lowery 
et al., 2014). The worsening of pain observed in this study could be related to increased PA or other aspects of health (Aarts et al., 2015). This result indicates that lung cancer survivors involved in PA interventions could require more careful follow-up. Given the current findings of increased pain following the intervention, a more thorough analysis of pain (e.g., including multi-faceted measures of pain like the Brief Pain Inventory) should be considered to elucidate the nature and correlates of pain experienced in this setting.

This is a feasibility study with associated limitations. The small sample size and lack of control group limit the conclusions that can be drawn from this work. The study suffered from a low response rate; therefore, the results may not be representative of the target population. In fact, we originally planned to undertake a randomised controlled trial, but it became clear that the number of participants approachable from the state cancer registry was too small to implement such a design. We suggest that future research should consider using multiple recruitment techniques (e.g., recruitment through clinics at the end of treatment when patients might be more open to receiving a health-behaviour intervention) and multi-site interventions to increase sample size. Another consideration is that the intervention is not appropriate for lung cancer survivors with complicated or severe comorbidity profiles who would require more thorough pre-intervention screening than is available for a distance-based intervention (e.g., supervised exercise testing). Given the high rates of comorbidity in this population, this could impact the generalisability of our intervention, although it only accounted for $6 \%$ of those ineligible in this study. Although telephone follow-up was designed to be short (i.e., between 5 and $10 \mathrm{~min}$ ), future research should consider other strategies to reduce the length of calls, or vary the schedule to reduce burden (e.g., biweekly calls rather than weekly calls in first 4 weeks).

In light of our lack of maintenance effects of the intervention, future research could consider increasing the intervention period and perhaps continuation of a lower level of intervention for potential maintenance of benefit. In this study, the intervention was based on the Theory of Planned Behavior and included materials focused on implementation intentions, or specifying when, where and how of goal setting and overcoming barriers, as well as self-efficacy and perceived controllability. Targeting these constructs has been shown to assist with initiating behaviour change in clinical populations (Teixeira et al., 2015). However, supporting maintenance of behavioural change during follow-up periods could benefit from different theoretical perspectives. As one example, using a Social Ecological Model approach could allow a broader perspective of multilevel interventions, that can focus beyond individual-level components such as the techniques employed here, to add interpersonal (e.g., group education sessions), and community-level components (e.g., tailored walking maps) to support behaviour maintenance (Kerr et al., 2012).

All of the outcome measures employed in this study are self-report. There are significant limitations in using self-report measures of PA including over- and under-estimation of true PA participation as well as rates of inactivity (Prince et al., 2008). Self-reported PA results are subject to over-reporting and social desirability bias (Sallis \& Saelens, 2000), and this must be considered when evaluating these results. The costs and benefits of objective measurement of PA need to be considered in any study (Prince et al., 2008), and the pilot nature of this work in a distance-based setting, paired with a limited budget precluded objective measures of PA. However, using an objective measure of PA would have provided better quantification of PA volume and intensity as well as assessing sedentary time and should be strongly considered for future research.

The results of this study should be extended in the future by employing research designs, such as randomised controlled trials, that are able to assess the intervention in comparison to standard care. In addition, other techniques to improve compliance and retention, such as technology (e.g., apps, wearable technology, text reminders), could improve retention of participants and behaviour maintenance. Finally, home-based interventions designed to target different modalities of exercise that could address the functional limitations experienced by this patient population, such as resistance exercise, could be a valuable avenue for future research.

\section{5 | CONCLUSION}

Our findings suggest that this pragmatic distance-based intervention was safe, had good adherence rates and indicate potential for improving short-term PA and QoL in lung cancer survivors. However, additional strategies are needed to improve other indicators of feasibility, particularly recruitment and retention of participants, as well as longterm maintenance of improvements.

\section{REFERENCES}

Aarts, M. J., Aerts, J. G., van den Borne, B. E., Biesma, B., Lemmens, V. E., \& Kloover, J. S. (2015). Comorbidity in patients with small-cell lung cancer: Trends and prognostic impact. Clinical Lung Cancer, 16, 282-291.

Ajzen, I. (1991). The theory of planned behavior. Organizational Behavior and Human Decision Processes, 50, 179-211.

Arem, H., Pfeiffer, R. M., Engels, E. A., Alfano, C. M., Hollenbeck, A., Park, Y., \& Matthews, C. E. (2015). Pre- and postdiagnosis physical activity, television viewing, and mortality among patients with colorectal cancer in the National Institutes of Health-AARP Diet and Health Study. Journal of Clinical Oncology, 33, 180-188.

Armstrong, T., Bauman, A., \& Davies, J. (2000). Physical activity patterns of Australian adults. Results of the 1999 national physical activity survey. Canberra, ACT: Australian Institute of Health and Welfare.

Balduyck, B., Hendriks, J., Lauwers, P., \& van Schil, P. (2007). Quality of life evolution after lung cancer surgery: A prospective study in $100 \mathrm{pa}-$ tients. Lung Cancer, 56, 423-431.

Bennett, J. A., Lyons, K. S., Winters-Stone, K., Nail, L. M., \& Scherer, J. (2007). Motivational interviewing to increase physical activity in longterm cancer survivors: A randomized controlled trial. Nursing Research, 56, 18-27.

Brown, J. K., Byers, T., Doyle, C., Coumeya, K. S., Demark-Wahnefried, W., Kushi, L. H., ... Sawyer, K. A. (2003). Nutrition and physical activity during and after cancer treatment: An American Cancer Society guide for informed choices. CA: A Cancer Journal for Clinicians, 53, 268-291.

Brunelli, A., Socci, L., Refai, M., Salati, M., Xiume, F., \& Sabbatini, A. (2007). Quality of life before and after major lung resection for lung cancer: $A$ prospective follow-up analysis. Annals of Thoracic Surgery, 84, 410-416.

Cavalheri, V., Tahirah, F., Nonoyama, M., Jenkins, S., \& Hill, K. (2014). Exercise training for people following lung resection for non-small cell 
lung cancer - A Cochrane systematic review. Cancer Treatment Reviews, 40, 585-594.

Cella, D. F., Bonomi, A. E., Lloyd, S. R., Tulsky, D. S., Kaplan, E., \& Bonomi, P. (1995). Reliability and validity of the Functional Assessment of Cancer Therapy-Lung (FACT-L) quality of life instrument. Lung Cancer, 12, 199-220.

Cella, D., Eton, D. T., Lai, J. S., Peterman, A. H., \& Merkel, D. (2002). Combining anchor and distribution-based methods to derive minimal clinically important differences on the Functional Assessment of Cancer Therapy (FACT) anemia and fatigue scales. Journal of Pain and Symptom Management, 24, 547-561.

Chen, H. M., Tsai, C. M., Wu, Y. C., Lin, K. C., \& Lin, C. C. (2015). Randomised controlled trial on the effectiveness of home-based walking exercise on anxiety, depression and cancer-related symptoms in patients with lung cancer. British Journal of Cancer, 112, 438-445.

Coups, E. J., Park, B. J., Feinstein, M. B., Steingart, R. M., Egleston, B. L., Wilson, D. J., \& Ostroff, J. S. (2009a). Correlates of physical activity among lung cancer survivors. Psychooncology, 18, 395-404.

Coups, E. J., Park, B. J., Feinstein, M. B., Steingart, R. M., Egleston, B. L., Wilson, D. J., \& Ostroff, J. S. (2009b). Physical activity among lung cancer survivors: Changes across the cancer trajectory and associations with quality of life. Cancer Epidemiology, Biomarkers \& Prevention, 18, 664-672.

Demark-Wahnefried, W., Clipp, E. C., Lipkus, I. M., Lobach, D., Snyder, D. C., Sloane, R., ... Kraus, W. E. (2007). Main outcomes of the FRESH START trial: A sequentially tailored, diet and exercise mailed print intervention among breast and prostate cancer survivors. Journal of Clinical Oncology, 25, 2709-2718.

Demark-Wahnefried, W., Clipp, E. C., Morey, M. C., Pieper, C. F., Sloane, R., Snyder, D. C., \& Cohen, H. J. (2006). Lifestyle intervention development study to improve physical function in older adults with cancer: Outcomes from Project LEAD. Journal of Clinical Oncology, 24, 3465-3473.

Denlinger, C. S., Carlson, R. W., Are, M., Baker, K. S., Davis, E., Edge, S. B., ... Freedman-Cass, D. (2014). Survivorship: Introduction and definition. Clinical practice guidelines in oncology. Journal of the National Comprehensive Cancer Network, 12, 34-45.

Ferlay, J., Soerjomataram, I., Dikshit, R., Eser, S., Mathers, C., Rebelo, M., ... Bray, F. (2015). Cancer incidence and mortality worldwide: Sources, methods and major patterns in GLOBOCAN 2012. International Journal of Cancer, 136, E359-E386.

Gester, F., Paulus, A., Sibille, A. L., Duysinx, B., \& Louis, R. (2016). Prognostic factors in non small cell lung cancer. Revue Medicale de Liege, 71, 34-39.

Godin, G., Jobin, J., \& Bouillon, J. (1986). Assessment of leisure time exercise behavior by self-report: A concurrent validiy study. Canadian Journal of Public Health, 77, 359-361.

Goode, A. D., Lawler, S. P., Brakenridge, C. L., Reeves, M. M., \& Eakin, E. G. (2015). Telephone, print, and web-based interventions for physical activity, diet, and weight control among cancer survivors: A systematic review. Journal of Cancer Survivorship, 9, 660-682.

Granger, C. L., Denehy, L., McDonald, C. F., Irving, L., \& Clark, R. A. (2014). Physical activity measured using global positioning system tracking in non-small cell lung cancer: An observational study. Integrative Cancer Therapy, 13, 482-492.

Granger, C. L., McDonald, C. F., Irving, L., Clark, R. A., Gough, K., Murnane, A., ... Denehy, L. (2014). Low physical activity levels and functional decline in individuals with lung cancer. Lung Cancer, 83, 292-299.

Gunnell, A. S., Einarsdottir, K., Galvao, D. A., Joyce, S., Tomlin, S., Graham, V., ... Briffa, T. (2013). Lifestyle factors, medication use and risk for ischaemic heart disease hospitalisation: A longitudinal population-based study. PLoS ONE, 8, e77833.

Handy, J. R., Asaph, J. W., Skokan, L., Reed, C. E., Koh, S., Brooks, G., ... Silvestri, G. A. (2002). What happens to patients undergoing lung cancer surgery? Outcomes and quality of life before and after surgery. Chest, 122, 21-30.
Hatchett, A., Hallam, J. S., \& Ford, M. A. (2013). Evaluation of a social cognitive theory-based email intervention designed to influence the physical activity of survivors of breast cancer. Psychooncology, 22, 829-836.

Jenkinson, C., Wright, L., \& Coulter, A. (1994). Criterion validity and reliability of the SF-36 in a population sample. Quality of Life Research, 3, 7-12.

Kerr, J., Rosenberg, D. E., Nathan, A., Millstein, R. A., Carlson, J. A., Crist, K., ... Marshall, S. J. (2012). Applying the ecological model of behavior change to a physical activity trial in retirement communities: Description of the study protocol. Contemporary Clinical Trials, 33, 1180-1188.

Khuri, F. R., McKenna, R. J., \& Movas, B. (2000). Non-small cell lung cancer. In R. Paxdur, J.R. Coia, W.J. Hoskins \& L.D. Wagman (Eds.), Cancer management: A multidisciplinary approach. Melville, NY: PRR.

Lakoski, S. G., Eves, N. D., Douglas, P. S., \& Jones, L. W. (2012). Exercise rehabilitation in patients with cancer. Nature Reviews. Clinical Oncology, 9, 288-296.

Leach, C. R., Bellizzi, K. M., Hurria, A., \& Reeve, B. B. (2016). Is it my cancer or am I just getting older? Impact of cancer on age-related health conditions of older cancer survivors. Cancer, 122, 1946-1953.

Leach, H. J., Devonish, J. A., Bebb, D. G., Krenz, K. A., \& Culos-Reed, S. N. (2015). Exercise preferences, levels and quality of life in lung cancer survivors. Support Care Cancer. doi: 10.1007/s00520-015-2717-6.

Ligibel, J. A., Meyerhardt, J., Pierce, J. P., Najita, J., Shockro, L., Campbell, N., ... Shapiro, C. (2012). Impact of a telephone-based physical activity intervention upon exercise behaviors and fitness in cancer survivors enrolled in a cooperative group setting. Breast Cancer Research and Treatment, 132, 205-213.

Lowery, A. E., Krebs, P., Coups, E. J., Feinstein, M. B., Burkhalter, J. E., Park, B. J., \& Ostroff, J. S. (2014). Impact of symptom burden in post-surgical non-small cell lung cancer survivors. Supportive Care in Cancer, 22, 173-180.

Lyons, R. A., Perry, H. M., \& Littlepage, B. N. (1994). Evidence for the validity of the Short-form 36 Questionnaire (SF-36) in an elderly population. Age and Ageing, 23, 182-184.

McCahon, D., Daley, A. J., Jones, J., Haslop, R., Shajpal, A., Taylor, A., ... Dowswell, G. (2015). Enhancing adherence in trials promoting change in diet and physical activity in individuals with a diagnosis of colorectal adenoma: A systematic review of behavioural intervention approaches. BMC Cancer, 15, 505.

Ogle, K. S. K. (2000). Cancer and comorbidity: Redefining chronic diseases. Cancer, 88, 653-663.

Peddle, C. J., Jones, L. W., Eves, N. D., Reiman, T., Sellar, C. M., Winton, T., \& Courneya, K. S. (2009). Correlates of adherence to supervised exercise in patients awaiting surgical removal of malignant lung lesions: Results of a pilot study. Oncology Nursing Forum, 36, 287-295.

Peddle-Mcintyre, C. J., Bell, G., Fenton, D., McCargar, L., \& Courneya, K. S. (2012). Feasibility and preliminary efficacy of progressive resistance exercise training in lung cancer survivors. Lung Cancer, 75, 126-132.

Peddle-Mcintyre, C. J., Bell, G., Fenton, D., McCargar, L., \& Courneya, K. S. (2013). Changes in motivational outcomes after a supervised resistance exercise training intervention in lung cancer survivors. Cancer Nursing, 36, E27-E35.

Philip, E. J., Coups, E. J., Feinstein, M. B., Park, B. J., Wilson, D. J., \& Ostroff, J. S. (2014). Physical activity preferences of early-stage lung cancer survivors. Supportive Care in Cancer, 22, 495-502.

Pinto, B. M., Frierson, G. M., Rabin, C., Trunzo, J. J., \& Marcus, B. H. (2005). Home-based physical activity intervention for breast cancer patients. Journal of Clinical Oncology, 23, 3577-3587.

Pinto, B. M., Papandonatos, G. D., Goldstein, M. G., Marcus, B. H., \& Farrell, N. (2013). Home-based physical activity intervention for colorectal cancer survivors. Psychooncology, 22, 54-64.

Pinto, B. M., Rabin, C., Papandonatos, G. D., Frierson, G. M., Trunzo, J. J., \& Marcus, B. H. (2008). Maintenance of effects of a home-based physical activity program among breast cancer survivors. Supportive Care in Cancer, 16, 1279-1289. 
Prince, S. A., Adamo, K. B., Hamel, M. E., Hardt, J., Connor Gorber, S., \& Tremblay, M. (2008). A comparison of direct versus self-report measures for assessing physical activity in adults: A systematic review. International Journal of Behavioral Nutrition Physical Activity, 5, 56.

Rogers, L. Q., Markwell, S. J., Courneya, K. S., McAuley, E., \& Verhulst, S. (2011). Physical activity type and intensity among rural breast cancer survivors: Patterns and associations with fatigue and depressive symptoms. Journal of Cancer Survivorship, 5, 54-61.

Sallis, J. F., \& Saelens, B. E. (2000). Assessment of physical activity by selfreport: Status, limitations, and future directions. Research Quarterly for Exercise and Sport, 71(Suppl. 2), 1-14.

Schmitz, K. H., Courneya, K. S., Matthews, C., Demark-Wahnefried, W., Galvao, D. A., Pinto, B. M., ... Schwartz, A. L. (2010). American College of Sports Medicine roundtable on exercise guidelines for cancer survivors. Medicine and Science in Sports and Exercise, 42, 1409-1426.

Sellar, C. M., Bell, G. J., Haennel, R. G., Au, H. J., Chua, N., \& Courneya, K. S. (2014). Feasibility and efficacy of a 12 -week supervised exercise intervention for colorectal cancer survivors. Applied Physiology, Nutrition and Metabolism, 39, 715-723.

Tanaka, K., Akechi, T., Okuyama, T., Nishiwaki, Y., \& Uchitomi, Y. (2000). Development and validation of the Cancer Dyspnoea Scale: A multidimensional, brief, self-rating scale. British Journal of Cancer, 82, 800-805.

Teixeira, P. J., Carraca, E. V., Marques, M. M., Rutter, H., Oppert, J. M., de Bourdeaudhuij, I., ... Brug, J. (2015). Successful behavior change in obesity interventions in adults: A systematic review of self-regulation mediators. BMC Medicine, 13, 84.

Vallance, J. K., Courneya, K. S., Plotnikoff, R. C., \& Mackey, J. R. (2008). Analyzing theoretical mechanisms of physical activity behavior change in breast cancer survivors: Results from the activity promotion (ACTION) trial. Annals of Behavioral Medicine, 35, 150-158.
Vallance, J. K., Courneya, K. S., Plotnikoff, R. C., Yasui, Y., \& Mackey, J. R. (2007). Randomized controlled trial of the effects of print materials and step pedometers on physical activity and quality of life in breast cancer survivors. Journal of Clinical Oncology, 25, 2352-2359.

Vallance, J. K., Courneya, K. S., Taylor, L. M., Plotnikoff, R. C., \& Mackey, J. R. (2008). Development and evaluation of a theory-based physical activity guidebook for breast cancer survivors. Health Education and Behavior, 35, 174-189.

Vijayvergia, N., Shah, P. C., \& Denlinger, C. S. (2015). Survivorship in nonsmall cell lung cancer: Challenges faced and steps forward. Journal of the National Comprehensive Cancer Network, 13, 1151-1161.

Ware, J. E. Jr, Kosinski, M., Bjorner, J. B., Turner-Bowker, D. M., Gandek, B., \& Maruish, M. E. (2007). Unser's manual for the SF-36v2 health survey (2nd edn). Lincoln, RI, QualityMetric.

Yun, Y. H., Shon, E. J., Yang, A. J., Kim, S. H., Kim, Y. A., Chang, Y. J., ... Shim, Y. M. (2013). Needs regarding care and factors associated with unmet needs in disease-free survivors of surgically treated lung cancer. Annals of Oncology, 24, 1552-1559.

Zieren, H. U., Muller, J. M., Hamberger, U., \& Pichlmaier, H. (1996). Quality of life after surgical therapy of bronchogenic carcinoma. European Journal of Cardio-Thoracic Surgery, 10, 233-237.

How to cite this article: Peddle-Mclntyre CJ, Baker MK, Lee YCG, et al. The feasibility of a pragmatic distance-based intervention to increase physical activity in lung cancer survivors. Eur J Cancer Care. 2018;27:e12722. https://doi.org/10.1111/ecc.12722 